\title{
Blood transfusion management in the new era of immune therapy: experience from Dienst voor het Bloed, Rode Kruis-Vlaanderen
}

\author{
E. Lazarova, V. Pede, P. Gys, MP. Emonds, A. Muylaert, P. Vandekerckhove and V. Compernolle
}

\section{BACKGROUND}

Daratumumab (human monoclonal IgG1kappa that targets CD38) is already in use as monotherapy for adult patients with relapsed and refractory multiple myeloma (forth line therapy). Recently this medication received the extended approval as a second line therapy.

CD38 is the ecto-enzymic cyclic ADP ribose hydrolase that participate in regulation of intracellular $\mathrm{Ca} 2+$ and in receptor mediated adhesion. It is expressed on $\mathrm{B}, \mathrm{T}, \mathrm{NK}$, plasma cells and red blood cells (RBC), among many others.

As CD38 is also expressed on RBC, Daratumumab interferes with routine blood bank tests (antibody screening and identification, cross-match) by direct binding to CD38 expressed on reagent red blood cells (RBCs) or on cross-matched RBCs.

Various methods exist to eliminate or bypass the anti-CD38 effects:

- Dithiothreitol (DTT) or trypsin-treated reagent/cross-matched RBCs (denatures CD38, prevents Daratumumab binding; however, there are important DTT-denaturation sensitive blood group antigens)

- Cord RBCs (negligible CD38 expression, not commercialized)

- Anti-CD-38 neutralization (by anti-idiotype antibodies or soluble extracellular domain of CD38)

- Antigen-matched RBC transfusions guided by patient phenotyping or genotyping

Unfortunately, there is no consensus on the best method or combination of methods to be used.

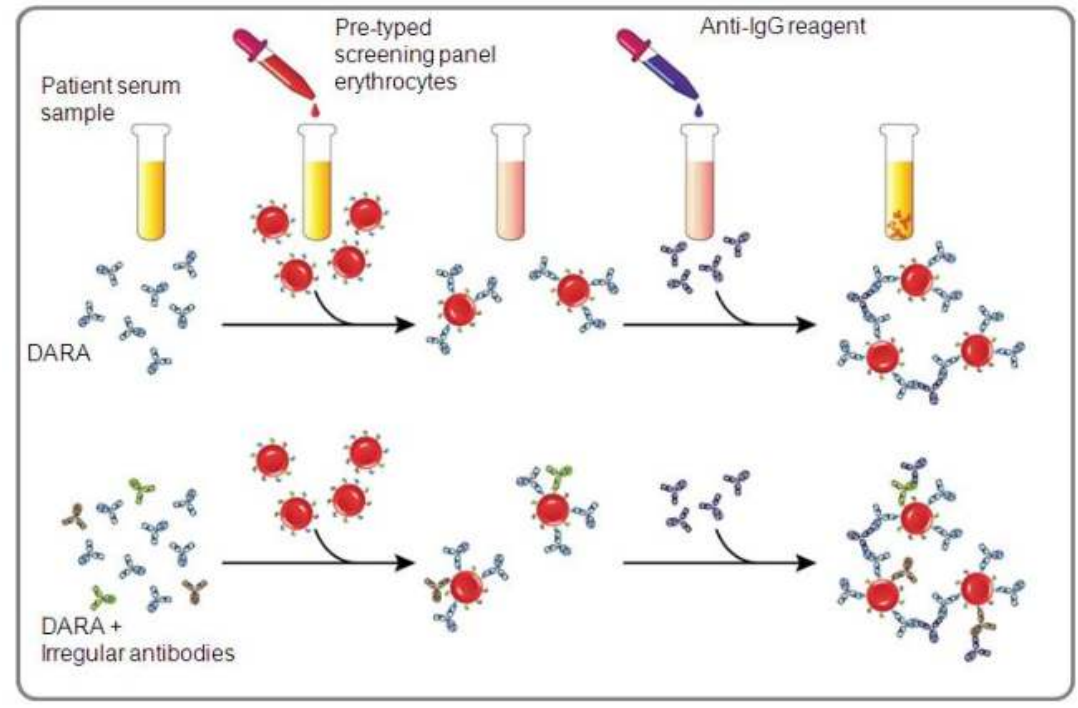

Daratumumab (DARA) in the patient's serum binds to the test RBCs. After adding the anti-IgG reagent, RBC agglutination is observed, thereby generating a false positive result. The presence of irregular antibodies is masked by the presence of DARA (Supporting information from Oostendorp et al, 2015)

\section{MATERIALS AND METHODS}

- One year study period (From November 2016 till November 2017)

- A total of $425 \mathrm{RBC}$ molecular typings were performed during that period in Dienst voor het Bloed, Rode Kruis-Vlaanderen

- PCR-SSP method with RBC-FluoGene vERYfy kit (Inno-Train) In the $\mathrm{Rh}$ system the $\mathrm{C}, \mathrm{c}, \mathrm{E}$, e and $\mathrm{Cw}$ alleles are detected. Furthermore typing of the blood groups Kell (KEL1/KEL2), Kidd (JK1/JK2), Duffy with the alleles FY1(A), FY2(B), FYX and Fynull (GATA-box mutation), MNS with the alleles MNS1(M), MNS2(N), MNS3(S), MNS4(s), U+var (P2) and $\mathrm{U}+\operatorname{var}(\mathrm{NY})$, as well as the Dombrock (DO1/DO2) are covered with this test kit

- Availability of an average of $24 \%$ of double phenotyped active donors from $\mathrm{O}$ of $\mathrm{A}$ blood group during this period

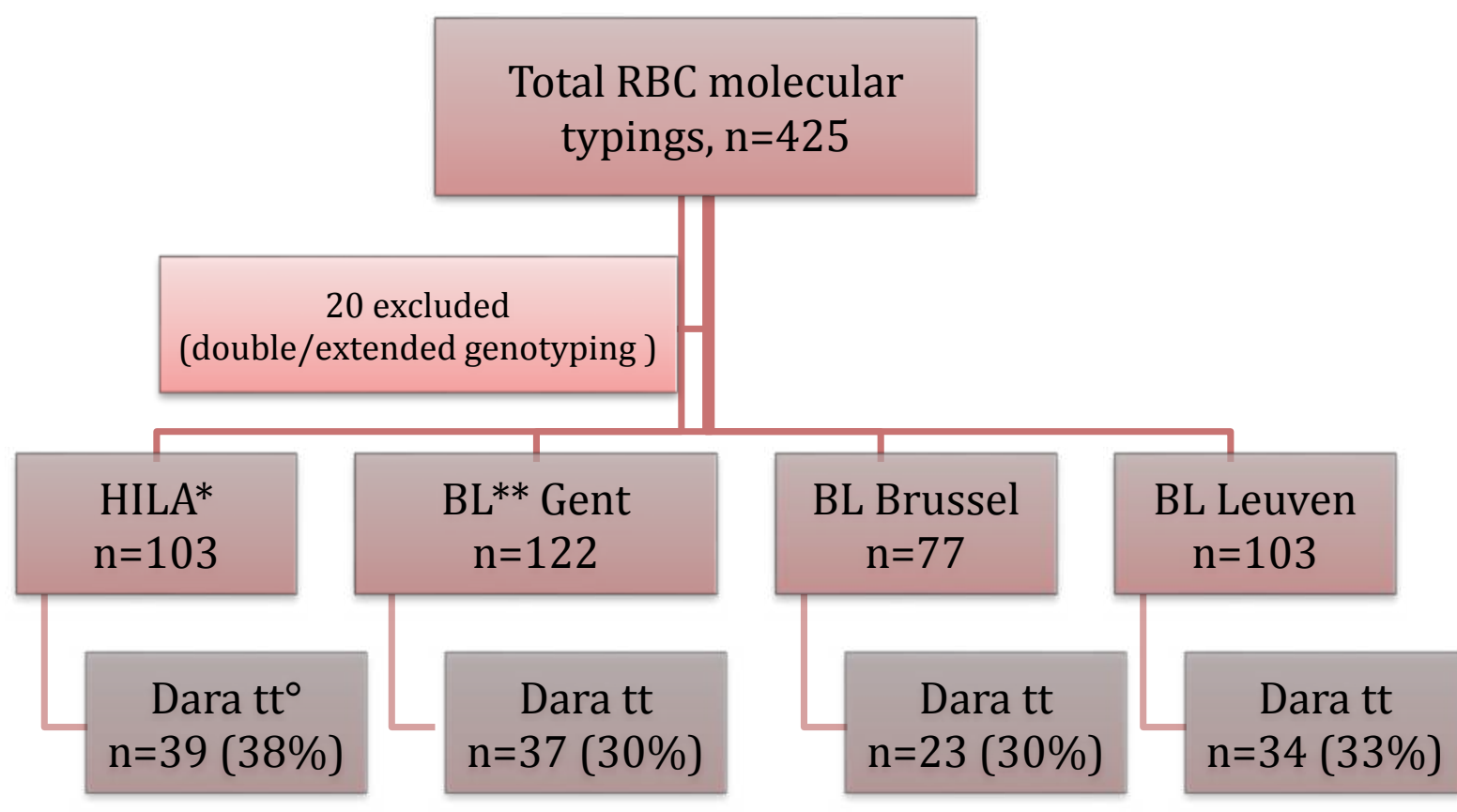

195 antigen-matched units $\rightarrow$ transfused to 27 patients

*HILA: Histocompatibility and immunogenetics laboratory

$* *$ BL: Bloodbank laboratory

${ }^{\circ}$ Dara tt: Patients under Daratumumab treatment

\section{RESULTS}

Rode Kruis-Vlaanderen (RKV) implemented the following transfusion policy in November 2016:

- RBC genotyping for clinically significant antigens is provided to all patients, ideally before starting therapy

- Afterwards, on demand, blood is selected on the basis of extended donor phenotype and patient's genetically predicted phenotype

- More than 130 patients enrolled in anti-CD38 treatment, were genotyped during the study period (32,8\% of all RBC genotypings)

- A total of 195 antigen-matched units were selected at RKV's blood bank laboratories and were transfused to a total of 27 patients

- An average of $24 \%$ of phenotyped active donors

- 25.640 available phenotyped blood group O RBCs

- 13.653 available phenotyped blood group A RBCs

- RKV is able to address actual blood transfusion need for these patients

- No adverse transfusion events and correct response to transfusions were reported.

\section{CONCLUSIONS}

This approach avoids time and labor consuming pre-transfusion serologic techniques for CD38 elimination. Delay in issuing of RBC units is prevented too.

If in the future Daratumumab becomes more widely used, the availability of convenient laboratory tests to cross match RBCs may become critical for adequate blood supply.

The selection of antigen-matched RBCs to support transfusion decisions is already common practice in complex clinical situations with panreactivity (e.g. auto-immune hemolytic anemia).

Patients receiving Daratumumab could be successfully managed in the same manner. 